\title{
37. Bundeskongress mit Mitgliederversammlung
}

20. bis 23. September 2007 in Erfurt

Die Vorträge anlässlich der Eröffnungsveranstaltung im Bundesarbeitsgericht wie auch der anschließenden Tagung sind im Fokus dieses Heftes (S. 1 ff.) dokumentiert. Über die Mitgliederversammlung wurde bereits im Mitglieder-Newsletter vom letzten Dezember http://www.djb.de/mitgliederbereich/Newsletter-Mitglieder/ kurz berichtet, jeweils mit Verweisen auf die dazugehörigen Dokumente im Mitgliederbereich des djb-Homepage. Die Mitgliederversammlung hatte über einige Anträge des Bundesvorstands zu entscheiden, u. a. über die Beitragsordnung und den Wissenschafts- preis. Die beschlossene Beitrags- und die Preisordnung finden Sie in Gänze in diesem Heft abgedruckt. Im Hinblick auf die personellen Veränderungen im Bundesvorstand sind im Folgenden die beiden neu gewählten Vorstandsmitglieder porträtiert: die Vizepräsidentin, Vors. Richterin am OLG Brandenburg Ramona Pisal, und die zweite Beisitzerin im Bundesvorstand - zuständig für die Mitglieder in Ausbildung -, Anna Eberhard, Referendarin in Bonn. Auch Fotos gibt es natürlich, von denen Sie hier eine kleine Auswahl sehen. (AG)

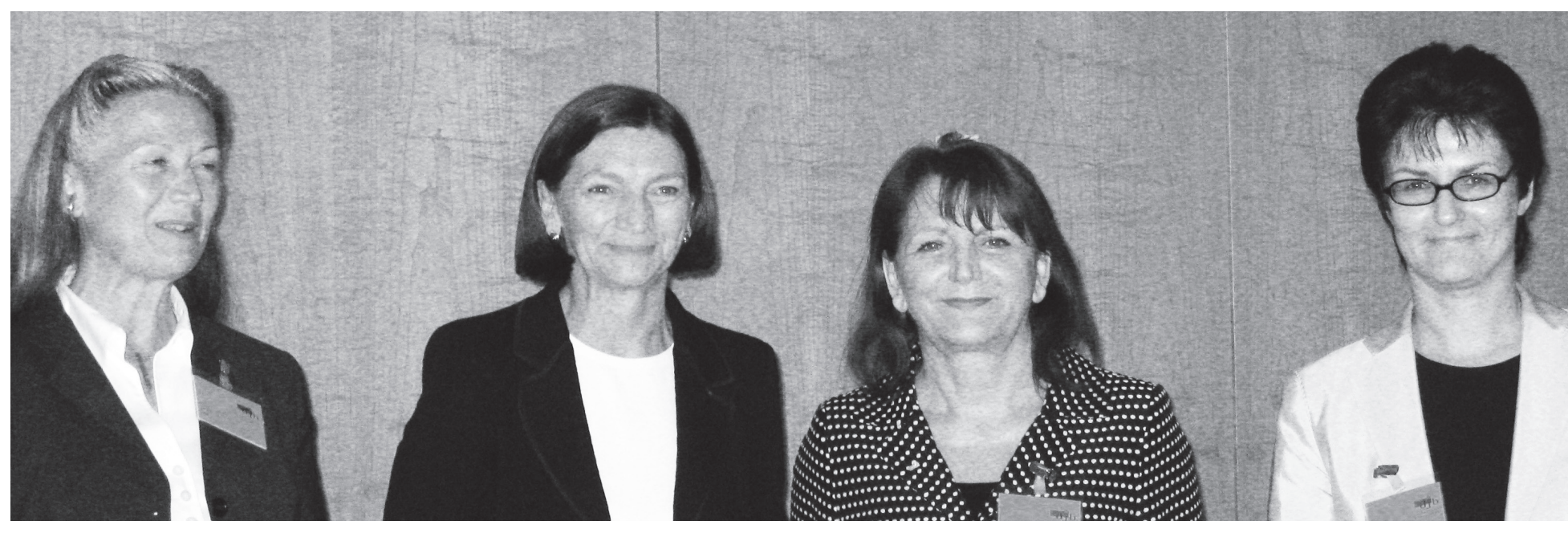

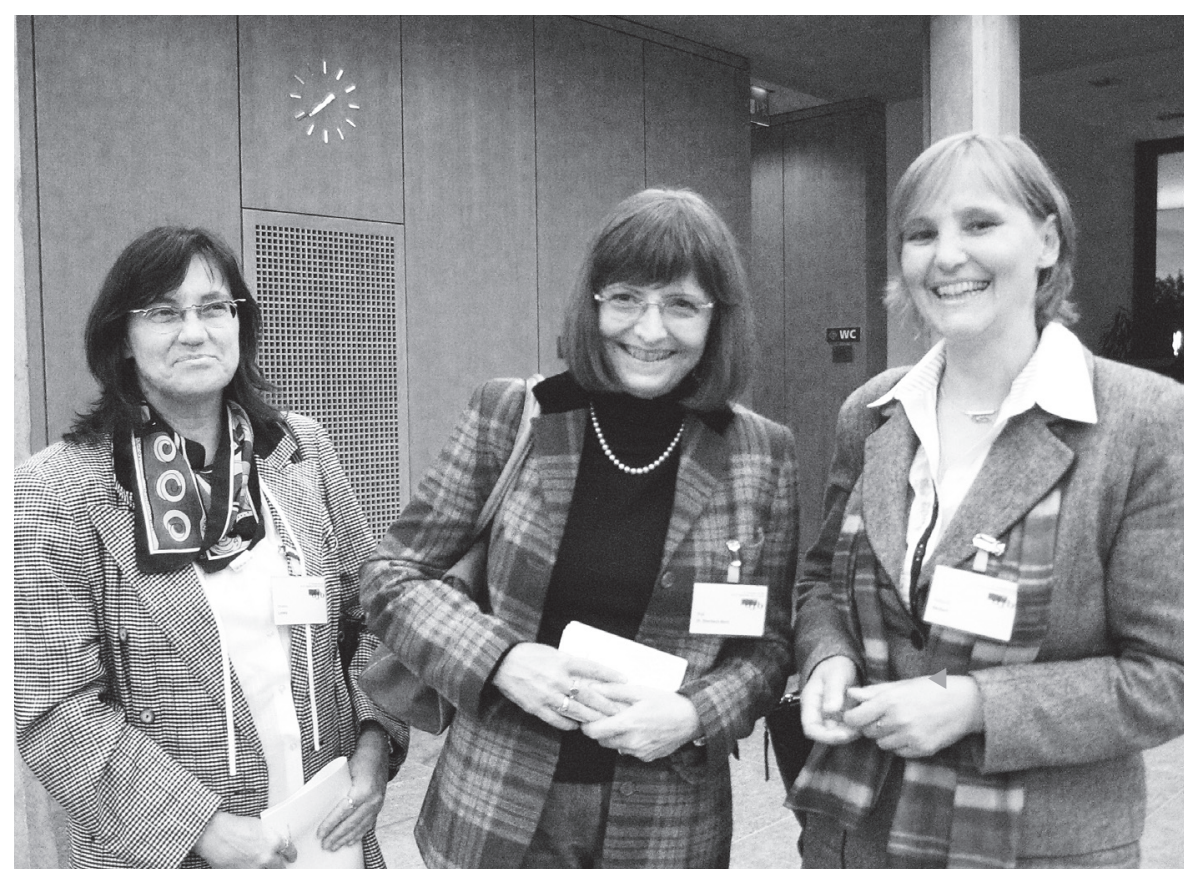

$\triangle$ Das neue Präsidium: Vizepräsidentin Ramona Pisal, Vors. Richterin am OLG Brandenburg; Präsidentin Jutta Wagner, Rechtsanwältin und Notarin in Berlin; Vizepräsidentin Renate Maltry, Rechtsanwältin in München; Schatzmeisterin Dagmar Brinkmann, Regierungsdirektorin beim Hess. Ministerium der Finanzen, Wiesbaden (v.I.n.r.). Foto: CL.

4 Christina Lorenz, Oberregierungsrätin im Thüringer Innenministerium, Vorsitzende des djbLandesverbands Thüringen; Dr. Birgit EberbachBorn, Direktorin beim Thüringer Landtag; Hildigund Neubert, Landesbeauftragte des Freistaates Thüringen für die Unterlagen des Staatssicherheitsdienstes der ehemaligen DDR (v.I.n.r.). Foto: $C D$. 


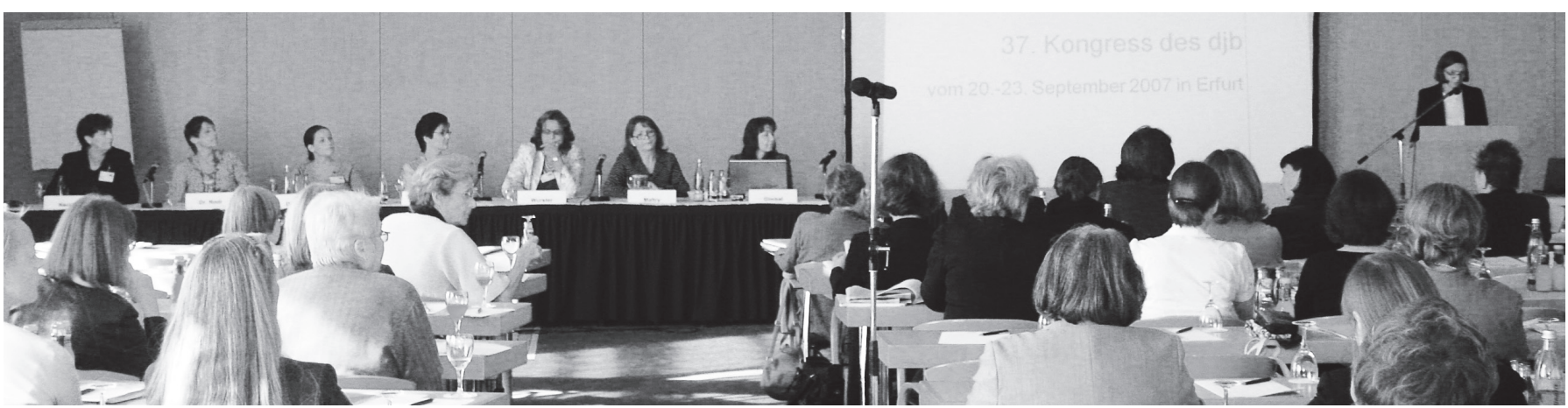

$\triangle$ Zu Beginn der Mitgliederversammlung am 22. September 2007 im Hotel Radisson SAS, Erfurt: Präsidentin Jutta Wagner berichtet über die Arbeit der vergangenen zwei Jahre. Foto: EH.
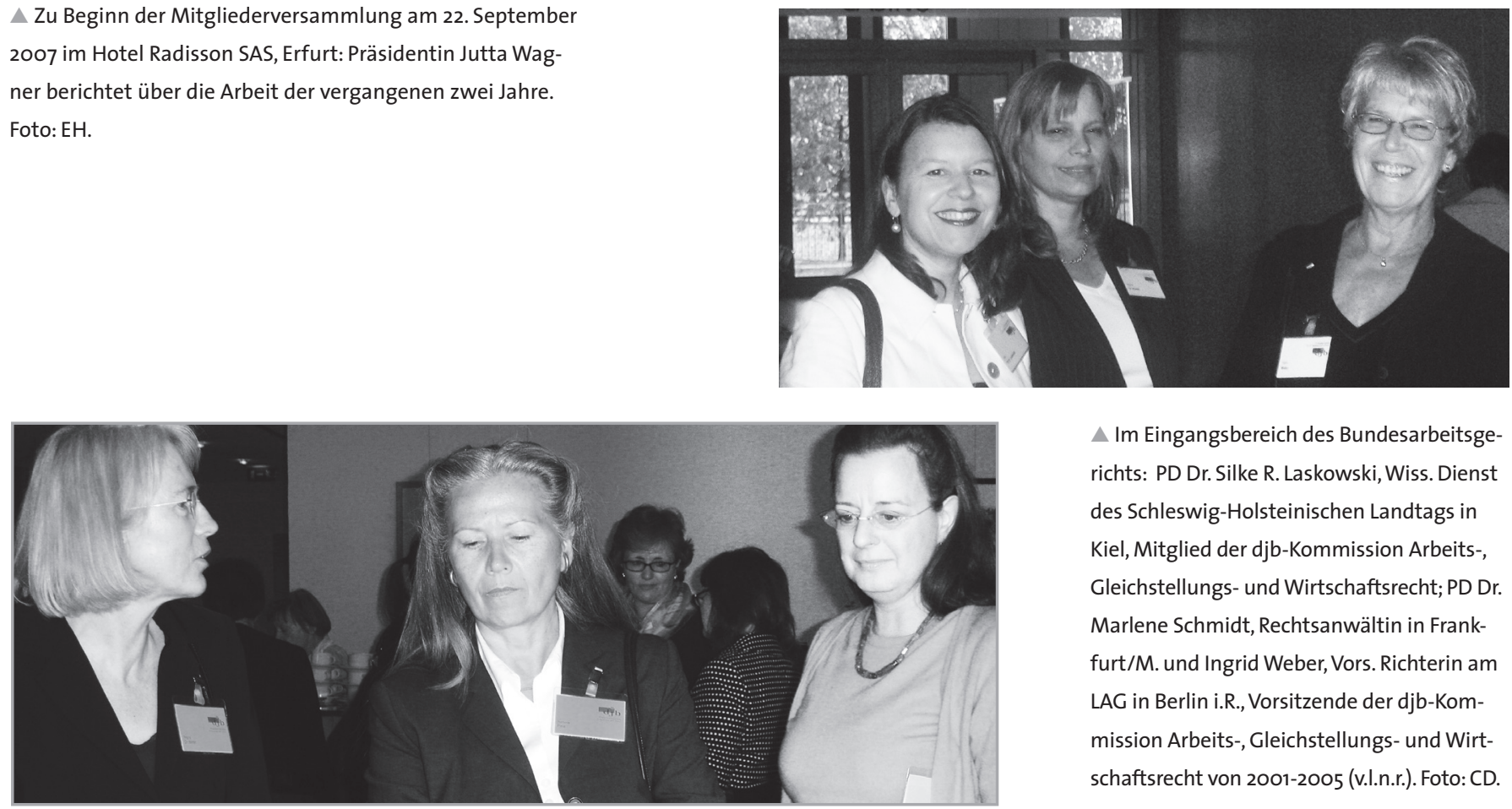

$\triangle$ Im Eingangsbereich des Bundesarbeitsgerichts: PD Dr. Silke R. Laskowski, Wiss. Dienst des Schleswig-Holsteinischen Landtags in Kiel, Mitglied der djb-Kommission Arbeits-, Gleichstellungs- und Wirtschaftsrecht; PD Dr. Marlene Schmidt, Rechtsanwältin in Frankfurt/M. und Ingrid Weber, Vors. Richterin am LAG in Berlin i.R., Vorsitzende der djb-Kommission Arbeits-, Gleichstellungs- und Wirtschaftsrecht von 2001-2005 (v.l.n.r.). Foto: CD.

$\triangle$ Zwischen den Vorträgen: Dr. Regine Winter, Rechtsreferentin am Gerichtshof der Europäischen Gemeinschaften in Straßburg, Mitglied der djb-Kommission Arbeits-, Gleichstellungs- und Wirtschaftsrecht; Ramona Pisal, Vors. Richterin am OLG Brandenburg, Vizepräsidentin des djb; Sabine Overkämping, Ministerialrätin im BMAS in Berlin, Leiterin der Untergruppe Europa in der djb-Kommission Öffentliches Recht, Europa- und Völkerrecht (v.l.n.r.). Foto: EH.

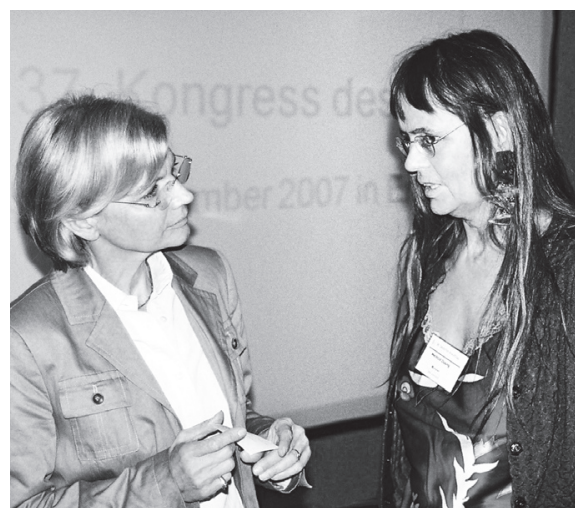

4 Dr. Karin Tondorf, freiberufliche Wissenschaftlerin und Beraterin, Forschung und Beratung zur Entgeltund Gleichstellungspolitik, Seddiner See, im Gespräch mit Mechtild Düsing, Rechtsanwältin in Münster, Vorsitzende der ARGE Anwältinnen im DAV. Foto: KS.

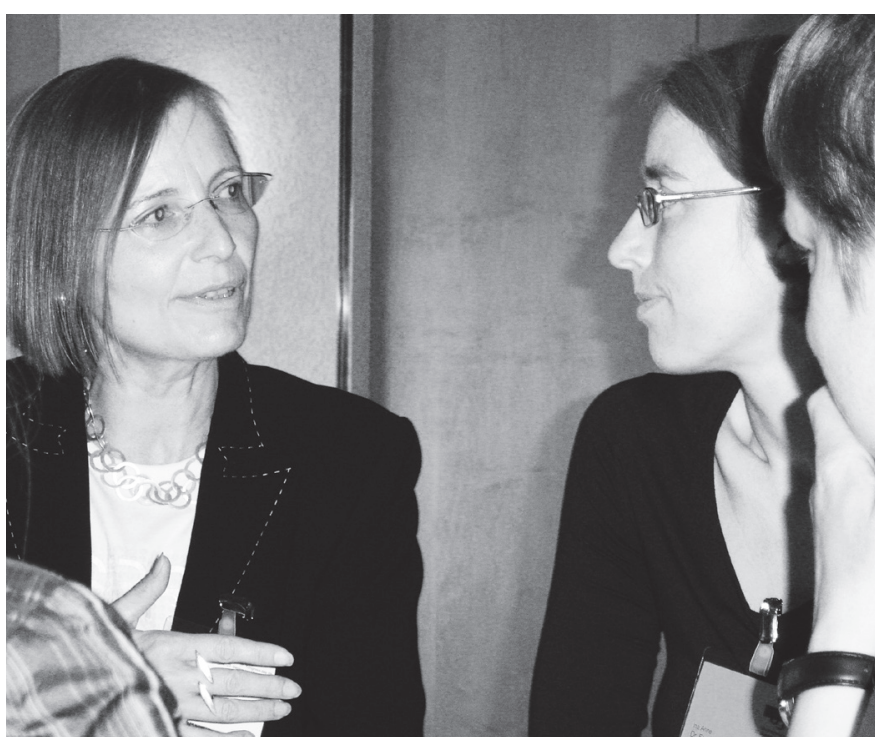

$\triangle$ Margarete Hofmann, Referatsleiterin in der EU-Kommission, Brüssel, Vors. der djb-RG Brüssel (links); Dr. Ina Anne Frost, Rechtsanwältin in Frankfurt/M. Foto: EH. 


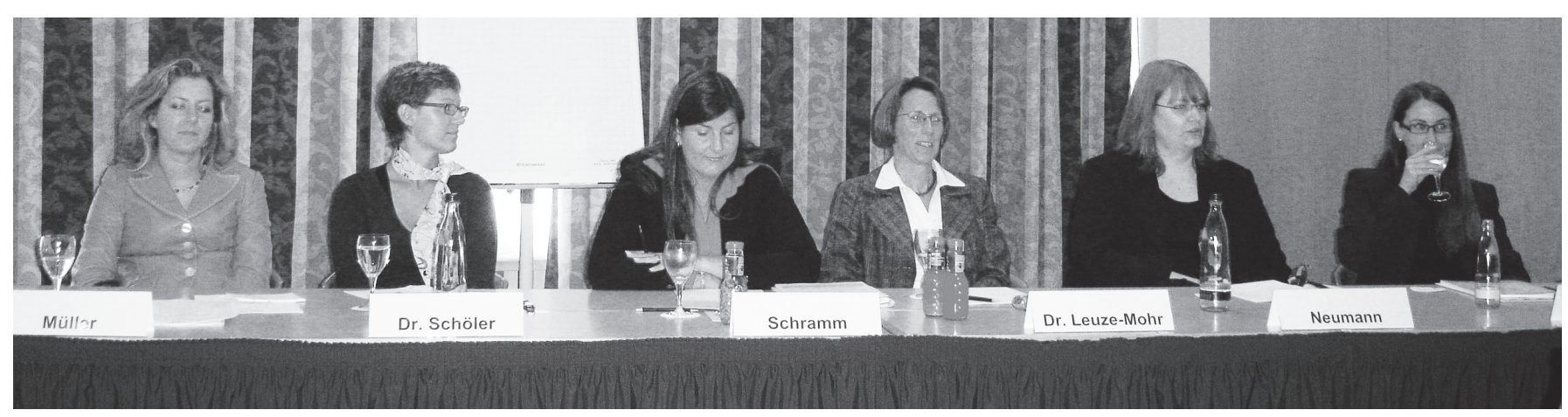

$\Delta$ Workshop “... und was, wenn ich nicht Anwältin oder Richterin werden will? - Alternative Berufsbilder im Recht" am 23. September 2007 in Erfurt. Foto: EH.

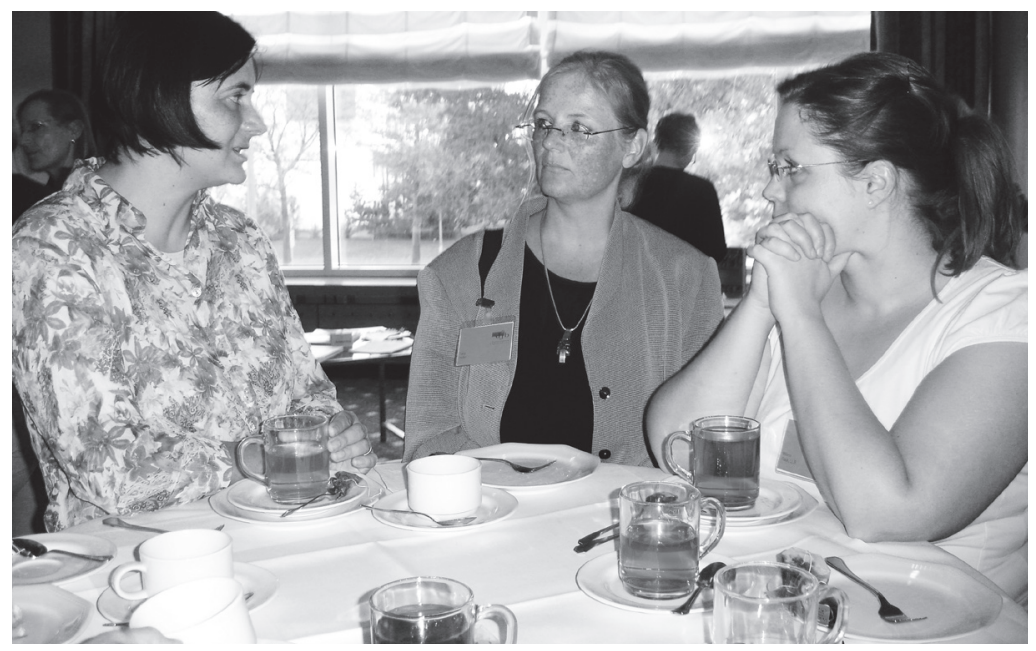

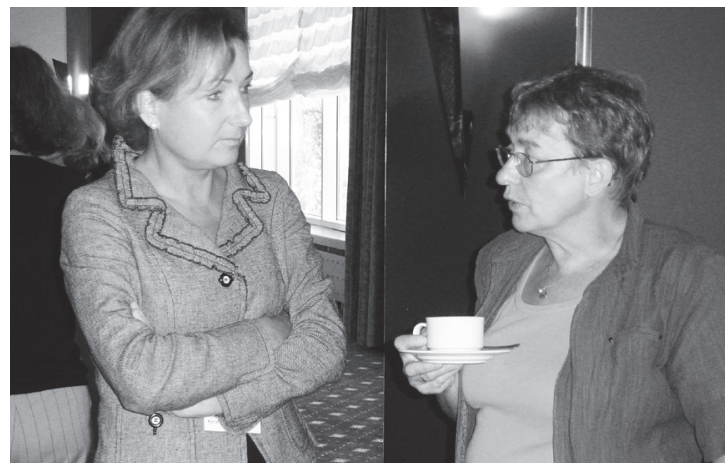

$\triangle$ Prof. Dr. Ulrike Mönig, Münster, Mitglied der djb-Kommission Gewalt gegen Frauen und Kinder (links); Dagmar Freudenberg, Staatsanwältin, Göttingen, Vors. der djb-Kommission Gewalt gegen Frauen und Kinder. Foto: $\mathrm{EH}$.

$\triangle$ Dr. Christine Fuchsloch, Richterin am LSG BerlinBrandenburg, Potsdam, Vorsitzende der Kommission Recht der sozialen Sicherung, Familienlastenausgleich von 2001-2005; Tanja Keller, Richterin am ArbG, München; Melanie Klauk, Rechtsreferendarin, Koblenz (v.I.n.r.). Foto: EH.

$\nabla$ Birgit Kersten, Rechtsanwältin und Vereidigte Buchprüferin in Brake/Unterweser, Vors. der djbRG Oldenburg (links); Anneliese Schmid-Kaufhold, Rechtsanwältin in Freiburg, Vors. der djb-RG Freiburg. Foto: $\mathrm{EH}$.

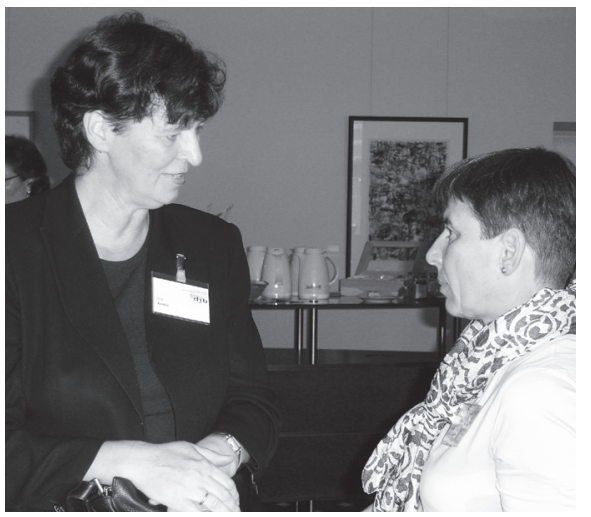

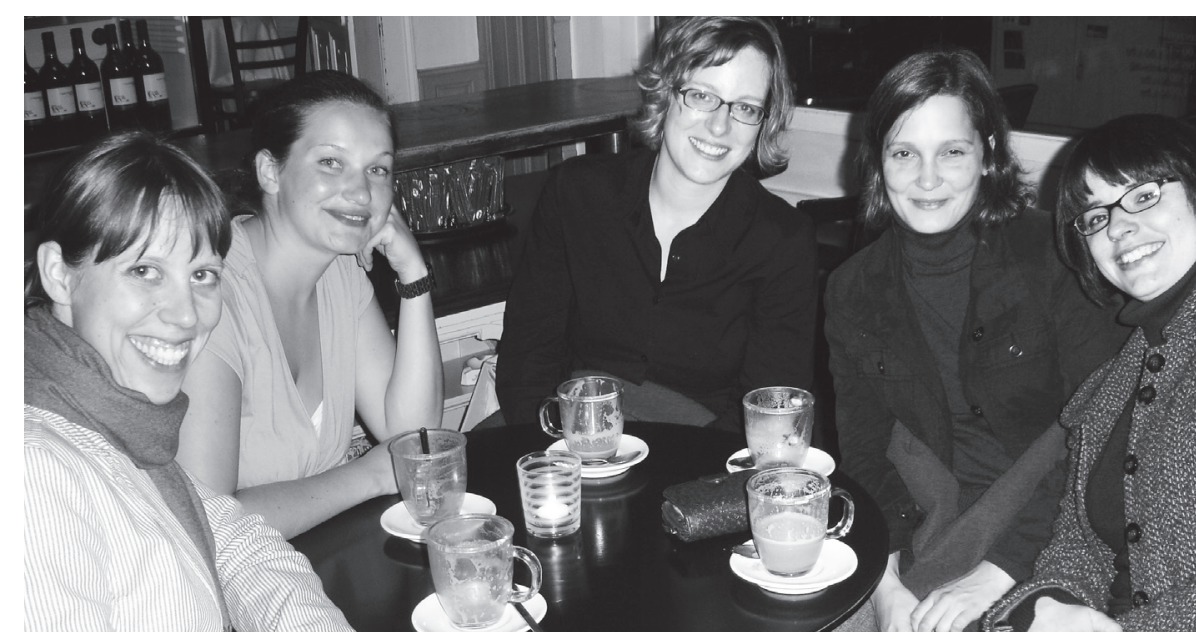

$\triangle$ Junge Juristinnen-Stammtisch in Erfurt: Anna Eberhard, Referendarin in Bonn und Barbara Dittmann, Referendarin in Koblenz, Beisitzerinnen im Bundesvorstand als Vertreterinnen der Mitglieder in Ausbildung; Dr. Diana Bartoszyk, Rechtsanwältin in Berlin; Vera Fischbach, Rechtsanwältin in Düsseldorf; Dr. Esther Hartwich, Assessorin, Berlin (v.l.n.r.). Foto: $\mathrm{EH}$. 